\title{
Female Hydrocele of Canal of Nuck: CT Findings
}

\author{
Kassem TW* \\ Department of Diagnostic and Interventional Radiology, \\ University of Cairo, Egypt \\ *Correspondling author: Kassem TW, Assistant \\ Professor Department of Diagnostic and Interventional \\ Radiology, University of Cairo, El-Manial Street, Cairo \\ University Hospitals (Kasr El Ainy), Faculty of Medicine, \\ Zip Code: 11956, Egypt
}

Received: September 28, 2017; Accepted: October 22, 2017; Published: October 30, 2017

\section{Abstract}

The canal of Nuck in females is formed of an invagination of parietal peritoneum that goes with the round ligament through the inguinal canal. Complete obliteration takes place during 1st year of life and its persistence result into hydrocele (female hydrocele) or hernia. Although it was thought to be extremely rare, nowadays it is diagnosed more frequently as physicians and radiologists became more familiar with this developmental disorder.

The current report presents a case of a 14-year-old girl complaining of slowly growing non painful swelling at the left inguinal region with no history of previous intervention. Post contrast multislice CT examination of the pelvis and inguinal region was requested aiming to define the extent and nature of the lesion. Multiplanar 2D and 3D images showed cystic lesion at the left inguinal canal having an intra pelvic component and midway constriction at the level of the internal inguinal ring providing accurate data for successful surgical planning.

Keywords: Female hydrocele; Canal of nuck; CT

\section{Introduction}

The canal of Nuck was first described in 1691 by Anton Nuck [1]. The saccus vaginalis of females is equivalent to process us vaginalis of males. Its extension into the inguinal canal is called "canal of Nuck". It is formed of an invagination of parietal peritoneum coming with the round ligament along its course from uterine cornu at proximal end till vulva at distal end [2]. Complete obliteration usually occurs during 1st year of life or it may persists and become liable to inguinal hernia occurrence [3]. Failure of obliteration may lead to cystic lesion formation "hydrocele of the canal of Nuck" [4]. The lining of parietal peritoneum secretes fluid. Cystic swelling takes place secondary to excessive secretions or slow absorption. This could be idiopathic or in consequence to trauma, inflammation or impaired lymphatic drainage [5].

\section{Case Presentation}

A 14-year-old girl complaining of slowly growing non painful swelling at the left inguinal region was referred to our practice. She had no history of previous intervention.

The examinations were performed using Aquilion 1 (320 channels) CT scanner (Toshiba Medical System, New York, USA), with tube potential set at $110 \mathrm{Kv}$, current at $320 \mathrm{~mA}$, collimation at 1 $\mathrm{mm}$ and table movement at $1 \mathrm{~mm} / \mathrm{s}$. A 14-gauge cannula was placed at a superficial vein at the ante cubital fossa, with the upper limbs placed over the head. Total amount of $100 \mathrm{ml}$ of non ionic contrast material (iopromide, $300 \mathrm{mg}$ iodine per ml, Ultravist 300; Schering AG, Berlin, Germany) was injected with an automatic injector at a flow rate of 2.5 $\mathrm{ml} / \mathrm{s}$. Acquisition began at the level of lower epigastrium till the upper thighs in portal phase.

An attached workstation and software were utilized to reconstruct axial source images into 2D multiplanar coronal / sagittal and 3D Volume Rendering (VR) images.

During interpretation of axial images a thin walled fluid filled structure was detected at the left inguinal region. It had an intra pelvic component related to the left lateral wall of the urinary bladder and extends distally along the left inguinal canal (Figure 1). It caused focal contour bulge of the overlying skin. There was no evidence of internal soft tissue densities or calcifications. The reconstructed coronal and sagittal images confirmed its cylindrical shape, oblique orientation and revealed a mid way constriction at the level of the left internal inguinal ring giving it the hour-glass appearance (Figure 2). It measured about $2.5 \times 2.1 \times 10.2 \mathrm{~cm}$ in transverse, antero-posterior and cranio-caudal diameters respectiely. The reconstructed 3D VR clearly identified the shape and location of the lesion (Figure 3).

\section{Discussion}

Cases of congenital hydrocele or hernia are commonly reported in male children and they are rare in females [6]. Incidence of hydrocele of canal of Nuck was reported by Huang et al. [7], to be $1 \%$ and Jagdale et al. [8] stated that associated inguinal hernia occur in one third of cases.

Clinically, it can appear as painless or mildly painful fluctuant inguinal swelling. It is not reducible and can be transilluminated [9].

Differential diagnosis of inguinal/labial swellings includes indirect inguinal hernia, hematoma, cold abscess, vascular aneurysm, cystic lymphangioma, lipoma, endometrioma, dermoid and ganglion cyst [10].

In literature, there is wide variety regarding age of incidence. While the current case is 14-year-old girl, similar diagnoses were reported in children 3-year-old [11], 7-year-old [12] as well as older patients 42-year old female [13] and 43-year old [9].

Contrast enhanced CT scan is the favored imaging modality for diagnosis of this pathology. A thin walled homogeneous fluid filled cyst is present [14]. Pandey et al. [13] used CT scan for diagnosis of their case as they reported multiloculated, hourglass-shaped cystic structure extending from left iliac region along inguinal canal to 


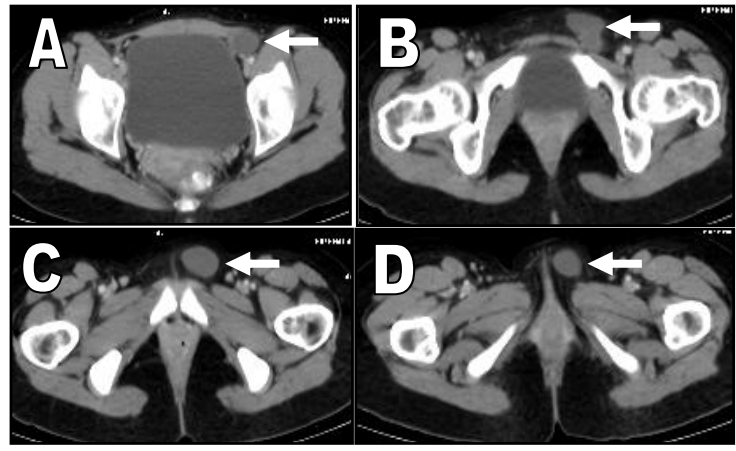

Figure 1: A, B, C and D: axial images for the pelvis and inguinal region showing the intra pelvic and inguinal components of left sided cylindrical cystic structure (arrows) with secondary contour bulge of the overlying skin.
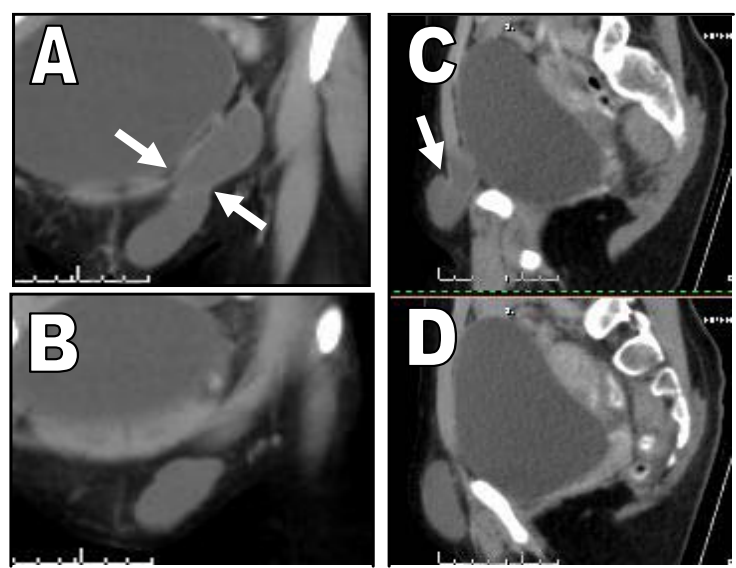

Figure 2: $A$ and $B$ : coronal images, $C$ and $D$ : sagittal images for the pelvis and inguinal region demonstrating the extent of the cystic lesion and midway constriction at the level of the left internal inguinal ring (arrows) giving the hour-glass appearance.

reach the labia majora. Additionally, a small rounded structure was found within the lesion that was confirmed by complementary US study to be a migrating ovary.

Park et al. [15] and Safak et al. [16] described MRI findings as thin walled cystic mass in inguinal having low intensity in T1 weighted images and high intensity in T2 weighted images with clear delineation of extension and location. Kono et al. [9] added MR hydrography technique with Maximum Intensity Projection (MIP) images for more accurate assessment. Ultrasound findings are a hypoechoic (or anechoic) sausage (or comma-shaped) superficial groin lesion medial to the pubic bone. It usually appears non vascular on Doppler study $[2,4,8]$.

Three types of hydrocele of canal of Nuck were reported. The first (most common type) has no communication with peritoneal cavity forming an encysted hydrocele from inguinal ring to vulva. The second type has persistent communication with peritoneal cavity. The third type represents a mix of first and second types. The inguinal ring constricts the hydrocele like a belt leaving a communicating part and an enclosed part (hourglass type) [13]. The current case was type III variety.

When the diagnosis is confirmed, surgical excision is the treatment

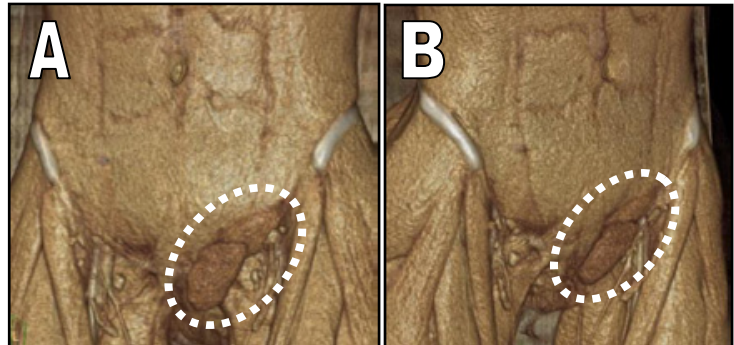

Figure 3: A and B: 3D Volume Rendering (VR) images clearly identifying the shape and location of the lesion (dashed circles).

of choice. There are no major post operative complications [12]. The current study was treated surgically after dissection and excision of the cyst and high ligation of peritoneal pouch neck. Laparoscopic approach can be used in management of hydrocele of canal of Nuck (type III) with associated inguinal hernia $[17,18]$.

\section{Conclusion}

When a female of any age presents with inguinal / labial swelling, diagnosis of hydrocele of canal of Nuck should be considered regardless its rarity. Contrast enhanced CT and/or MRI help to differentiate it from other possible swellings in same region and provide details facilitating surgical planning. The best line of treatment is complete surgical excision.

\section{References}

1. Tubbs RS, Loukas M, Shoja MM, Salter EG, Oakes WJ. Indirect inguinal hernia of the urinary bladder through a persistent canal of Nuck: case report. Hernia. 2007; 11: 287-288.

2. Anderson CC, Broadie TA, Mackey JE, Kopecky KK. Hydrocele of the canal of Nuck: ultrasound appearance. Am Surg. 1995; 61: 959-961.

3. Standring S. Grays Anatomy. $40^{\text {th }}$ edition. The anatomical basis of clinical practice. London: Elsevier Churchill Livingstone. 2005; 1321.

4. Manjunatha YC, Beeregowda YC, Bhaskaran A. Hydrocele of the canal of Nuck: imaging findings. Acta Radiologica Short Reports. 2012; 1:1-3.

5. Stickel WH, Manner M. Female Hydrocele (Cyst of the canal of Nuck) Sonographic Appearance of Rare and Little-Known Disorder. J Ultrasound Med. 2004; 23: 429-432.

6. Janssen K, Klinkner D, Kumar T. Encysted Hydrocele of Canal of Nuck: A Case Report with Review of Literature. Journal of Surgical Technique and Case Report. 2011; 3: 97-98.

7. Huang CS, Luo CC, Chao HC, Chu SM, Yu YJ, Yen JB. The presentation of asymptomatic palpable movable mass in female inguinal hernia. Eur $\mathrm{J}$ Pediatr. 2003; 162: 493-495.

8. Jagdale R, Agrawal S, Chhabra S, Jewan SY. Hydrocele of the Canal of Nuck: Value of Radiological Diagnosis. Journal of Radiology Case Reports. 2012; 6: 18-22.

9. Kono R, Terasaki H, Murakami N, Tanaka M, Takeda J, Abe T. Hydrocele of the canal of Nuck: a case report with magnetic resonance hydrography findings. Surgical Case Reports. 2015; 1: 86.

10. Jedrzejewski G, Stankiewicz A, Wieczorek AP. Uterus and ovary hernia of the canal of Nuck. Pediatr Radiol. 2008; 38: 1257-1258.

11. Sarkar S, Panja S, Kumar S. Hydrocele of the Canal of Nuck (Female Hydrocele): A Rare Differential for Inguino-Labial Swelling. Journal of Clinical and Diagnostic Research. 2016; 10: 21-22.

12. Rambhia S, Ayyar P. Hydrocele of canal of nuck: a case report. Int Surg J. 2015; 2: 396-397. 
13. Pandey A, Jain S, Verma A, Jain M, Srivastava A, Shukla R. Hydrocele of the canal of Nuck-Rare differential for vulval swelling. Indian Journal of Radiology and Imaging. 2014; 24: 175-177.

14. Chi YM, Lee GM, Yi JB, Yoon KL, Shin KS, Bae CW, et al. Two cases of female hydrocele of the canal of nuck. Korean J Pediatr. 2012; 55: 143-146.

15. Park SJ, Lee HK, Hong HS, Kim HC, Kim DH, Park JS, et al. Hydrocele of the canal of Nuck in a girl: ultrasound and MR appearance. Br J Radiol. 2004 77: 243-244.

16. Safak AA, Erdogmus B, Yazici B, Gokgoz AT. Hydrocele of the canal of Nuck: sonographic and MRI appearances. J Clin Ultrasound. 2007; 35: 531-532.
17. Matsumoto T, Hara T, Hirashita T, Kubo N, Hiroshige S, Orita H. Laparoscopic diagnosis and treatment of a hydrocele of the canal of Nuck extending in the retroperitoneal space: A case report. International Journal of Surgery Case Reports. 2014; 5: 861-864.

18. Qureshi NJ, Lakshman K. Laparoscopic excision of cyst of canal of Nuck. Journal of Minimal Access Surgery. 2014; 10: 87-89. 